250 particles with masses greater than $50 \mathrm{~g}$ should hit the Moon each day. Even going to the upper limit $(50,000 \mathrm{~g})$, of Duennebier et al.'s mass range the rate is about 10 per day. This compares with the maximum rate of 12 events per day observed by the seismic network. If meteoroid impacts are being detected the major meteor showers should stand out clearly above the sporadic background. The fact that Duennebier et al. are not seeing these showers introduces the possibility that they are seeing something else.

\section{Metals in proteins}

\section{from S. D. Dover}

THE environment of metals in metalloproteins is of growing interest with the advent of synchrotron radiation. As distinct from crystallographic studies, which reveal the whole molecule, X-ray absorption measurements in the neighbourhood of the absorption edge of a metal are indicative of the local environment.

The use of synchrotron radiation as a tool for studying that environment is probably at the beginning of its exponential growth phase. In this application a tunable source of $\mathrm{X}$ radiation is required so that the spectrum can be observed. Coventional tubes are designed to emit most of their X-ray energy in the characteristic radiation line. The white radiation background, for a similar wavelength spread, has a much lower intensity. This means that the power available from the synchrotron over a whole range of wavelengths is several orders of magnitude greater than from laboratory sources.

This factor makes it possible to measure in an acceptable time the Xray absorption spectra of systems where the absorber is relatively dilute (as in metalloproteins). Results from the Stanford Synchrotron Radiation Project (SPEAR) have been reported (Proc. natn. Acad. Sci. U.S.A., 72 , $2340 ; 1975)$ where gains in speed of $10^{4}-10^{5}$ over X-ray tubes have been obtained. For example, the spectrum for naemoglobin over a $400 \mathrm{eV}$ range was measured at $1 \mathrm{eV}$ steps in only $20 \mathrm{~h}$. This gave an acceptably low noise compared with the modulation of the $\mathrm{X}$-ray absorption.

It is the modulation of the absorption spectrum that is of interest. The spectrum of a free metal consists of a low absorption region below the absorption edge where the incident photon is insufficiently energetic to excite the $\mathrm{K}$ electron. With increasing energy of $\mathrm{X}$ rays, the absorption edge is reached as the $K$ electron can be excited to empty bound states. Beyond the edge itself the photon energy is sufficient to cause the emission of a photoelectron. The absorption tends to decrease monotonically from the absorption edge.

In contrast the spectrum of a bound metal in this region (the region of the extended X-ray absorption fine structure, EXAFS) is affected by the photoelectron being scattered back to the metal emitter by neighbouring atoms, inhibiting absorption. Instead of decreasing monotonically, the spectrum is modulated as the wavelength of the photoelectron varies with the wavelength of the X-ray photon. It is as if the photoelectron is acting as a diffraction probe for the environment of the metal. The back-scattering from surrounding atoms will have the appearance of interference fringes according to their distances from the metal and the photoelectron wavelength. Theoretical analysis of the fine structure of the spectrum has been successful in terms of the atomic environment for metals themselves ( $A d v$. $X$-ray Anal., 13, 248; 1970) and the iron-sulphur system in rubredoxin (Proc. natn. Acad. Sci. U.S.A., 72, 4003; 1975). Work is continuing on haemoglobin and metalloporphyrins (Proc. natn. Acad. Sci. U.S.A., 72, 2340; 1975) where the results from the porphyrins can be used in the interpretation of the environment of the haem iron.

The absorption edge itself is being studied in model compounds (Proc. natn. Acad. Sci. U.S.A., 73, 1384; 1976) where the fine structure and position is affected by the available bound electronic states. These and other model compound studies together with those of metalloproteins provide the possibility of examining the electronic situation of metals in proteins, and the two regions of the X-ray absorption spectrum together hold the promise of revealing the whole local environment.

\section{Fission of medium weight nuclei}

\section{from P. E. Hodgson}

THE fission of heavy nuclei such as uranium and plutonium is a well known phenomenon of practical importance. In special circumstances lighter nuclei can also undergo fission, and evidence for the fission of nuclei of mass around 80 has recently been found by Braun-Munzinger and colleagues (Phys. Rev. Lett., 36, 849; 1976).

The experiment giving evidence of such fission is the interaction of $140 \mathrm{MeV}^{32} \mathrm{~S}$ ions with ${ }^{50} \mathrm{Ti}$. This type of interaction has been studied for many years in order to determine the characteristics of heavy ion reactions, but the particular feature of the new experiment is an array of electronic counters that facilitates determination of the masses and energies of two of the products of the reaction. For

\section{The cytonet protein}

IN his report on the recent EMBO symposium, Bretscher (Nature, 261, 336; 1976) refers to the protein which Pearse (J. molec. Biol., 97, 93; 1975; Proc. natn. Acad. Sci. U.S.A., 73,1255 ; 1976) has shown to be associated with vesicle "coats". In separate publications on this same material in the biochemical and neuroanatomical literature, an important correlation appears to have gone unnoticed. Gray (J. Neurocytol., 1, 363; 1972; Brain Res., 62, 329; 1973) examined coated vesicles in presynaptic terminals in the central nervous system and concluded that the coat material is structurally indistinguishable from a fibrous network filling the intervesicular cytoplasm. He also noticed that coated vesicles are surrounded by a clear space which he interprets as a retraction zone caused by fracture of an extensive cytoplasmic network, part of which has artefactually collapsed onto the vesicle forming the coat. The coat then appears to be a distorted fragment of a diffuse yet discrete cytoplasmic organelle composed of a fibrous reticulum which Gray called the 'cytonet'.

Gray (J. Neurocytol., 4, 315; 1975) later repudiated not only vesicular coats but the entire cytonet structure as artefactual, thinking it to be composed of denatured protein complexes. However, Pearse's discovery that vesicular coats contain essentially one protein supports an interpretation of the cytonet as a distinct supramolecular structure. Since it pervades the cytoplasm of many cell types it seems likely to play some role in the spatial organisation of cyitoplasmic components, particularly of organelles such as synaptic vesicles. It thus seems important that the protein which coats isolated vesicles should be considered in its broader association with the cytonet, rather than in the limited context of "coated vesicles" which subcellular fraction studies imply. Pearse's proposed name for this protein (clathrin or "coat substance") is a misnomer. If the molecule must have a name 'cytonexin' would be better.

A. J. Matus 